\title{
Neighborhood Gradient Analysis : Examination of Variance in North Central Oklahoma Rainfall Statistics
}

\author{
Emmett J. Pybus and William L. Hughes \\ School of Electrical Engineering, Oklahoma State University, Stillwater 74074
}

(Manuscript received 30 April 1973, in revised form 4 September 1973)

\begin{abstract}
A method called Neighborhood Gradient Analysis is used to examine the variance in precipitation statistics for a neighborhood of Stillwater, Okla. The method uses: 1) radial geometry with respect to a "target" area (Stillwater), 2) yearly precipitation differences and gradients in a neighborhood of Stillwater, and 3) statistical behavior of the neighborhood over the years 1950-71. It is shown through use of correlation coefficients that year-to-year variation in precipitation contributes more to the variance statistic than does station-to-station variation during the same one year intervals. Using that information, it is shown, for example, that $10 \%$ increase (or lack of it) in Stillwater precipitation can be observed at a more than one standard deviation significance in three years' time.
\end{abstract}

\section{The problem}

The present state of the art of weather modification indicates that man has the ability to alter only a small percentage (up to $\pm 15 \%$ ) in annual precipitation. In any given year at any given station that amount of variation is much less than what one would expect from year to year under natural or unmodified conditions.

The statistical problems in general have been broadly discussed in the Fifth Berkeley Symposium series (1967). In that series of papers are summarized various attempts to experimentally alter the amount of precipitation with many and various types of controls for evaluation. The general consensus is that seeding is easy, evaluation is most difficult. Further and more recent discussion of the statistical problems is continued in Schickedanz and Huff (1971). A somewhat similar approach will be followed here. The notation and methods of Schickedanz and Huff $(\mathrm{S} \& \mathrm{H})$ will be followed closely in this paper, but several differences will be noted.

In comparing the $\mathrm{S} \& \mathrm{H}$ methods and location with the present work, three differences are apparent:

1) The locations are different, east-central Illinois being the $\mathrm{S} \& \mathrm{H}$ area of interest while central Oklahoma is the focus for this paper. Rainfall regimes are not identical for the two areas.

2) The present paper concerns itself with evaluation of an operational program and cannot plan nor carry out an experiment, as such. That is, there is presently no opportunity to plan nor implement additional sampling of rainfall or other parameters; no opportunity, nor desire, to randomize or select seeding opportunities; and no seeding discrimination between rainfall regimes. The experiment is subsidiary to the operation; therefore, learning is minimal. Results are to be maximal. A method for consumer testing of the service is, perhaps, the best description of this report.

3) Methods of analysis in this paper most closely resemble what $\mathbf{S} \& \mathbf{H}$ call the "target-control" method, but, in addition, the method of evaluation described here compares present continuous seeding behavior of target and control areas with historical behavior data for the same area. Thus, the method of this paper is not a clearly catagorized method of $S \& H$, but rather a combination of at least two of their methods.

Neyman et al. (1972) have pointed up the time influence and some of the geographic distribution problems in analyzing rainfall statistics from an Arizona cloud seeding experiment and others.

The present paper handles time increments of one year, thereby minimizing both the short-term $(24 \mathrm{hr})$ effects as well as minimizing the time-related information content of the results. On the other hand, this paper, as will be pointed out, specifically addresses the geographic distribution effects of weather modification in an Oklahoma region which minimizes orographic influence (contrary to most other experimental locations).

If the Neyman et al. findings also apply to this Oklahoma (plains) region, it may become necessary to extend the neighborhood gradient method to much further distances than previously thought.

\section{Reducing the complexities}

The method of Neighborhood Gradient Analysis reduces the variance in rainfall statistics as follows: 


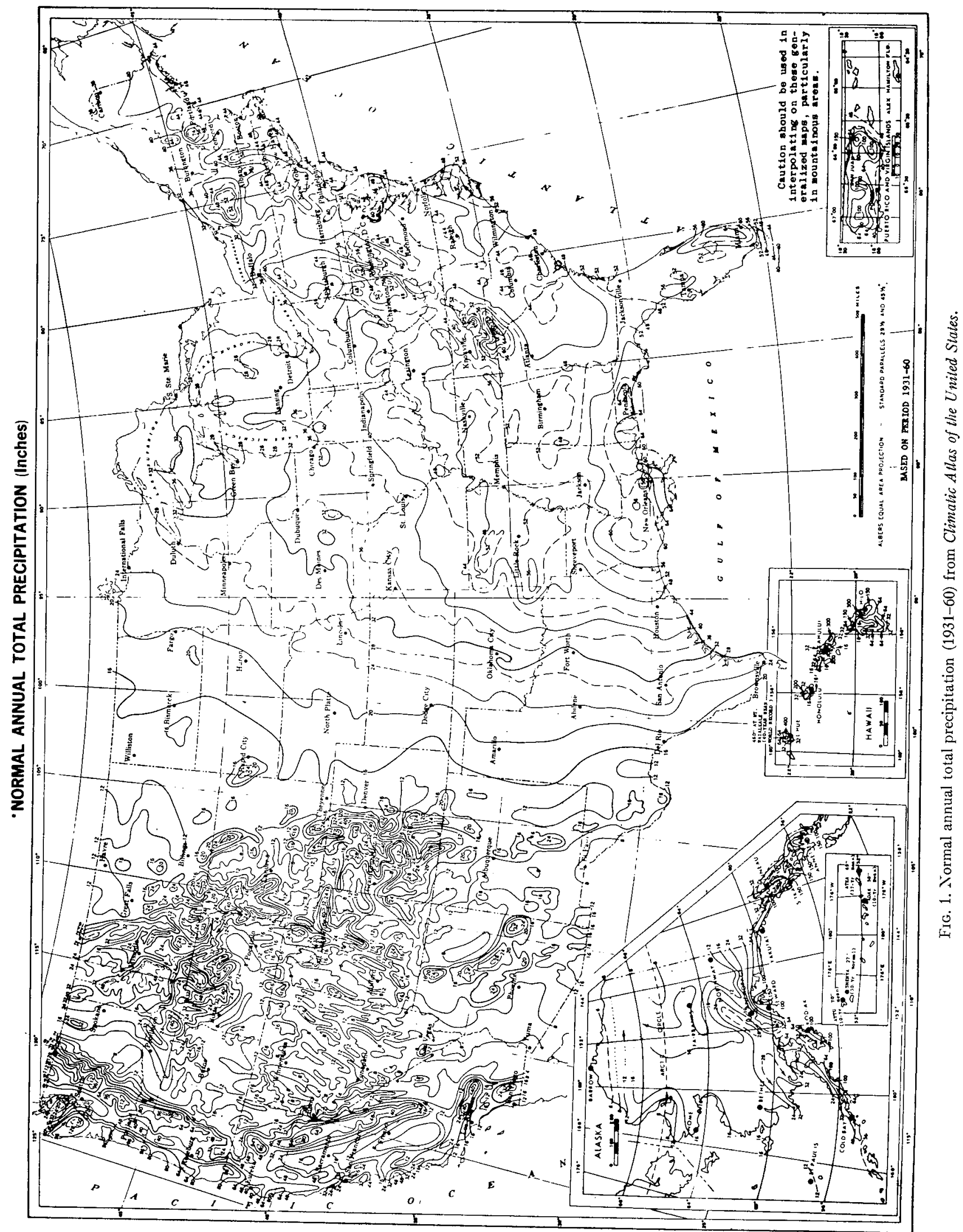


1) By regarding the targeted area as being imbedded in a substantially uniform meteorological situation. For example, if one looks at the U. S. Department of Commerce Climatic Atlas of the United States (p. 43) for normal annual total precipitation (shown in Fig. 1), there appears to exist a band of isohyets extending from the center of Texas northward through the Great Plains to Nebraska which are fairly regular in spacing, moderately flat in gradient, and smooth in contour over large areas. Any station within that area would seem to bear a fairly consistent and regular relationship to its surrounding neighbor stations.

2) By regarding the claims of absolute rainfall change as excessive zeal and, instead, viewing the modification of a target area as preferentially changing the gradient between that target and its surroundings during each available opportunity for modification. That is, the weather modification of concern is a percentage or relative change to a target with respect to the area outside that target and is to operate continuously.
3) By elimination, as much as possible, by choice of the area of study, orographic effects such as hills, mountain lee waves, or large sources of water. This is directly related to 1) in that the regularity of rainfall gradient also indicates regularity of topography and of meteorology.

4) By performing time domain analyses only after spatial or neighborhood differences and gradients are calculated. As implied in 1) and 2), geographic differences (spatial differences) should be of more importance in forming the basic data set than should time variations of the data at any one station.

Thus, we may simplify the statistical problem somewhat by stripping it down to a gradient (spatial) analysis imbedded in a regular, predictable setting involving only air mass (including frontal and convective but minimizing orographic) meteorology.

The uniqueness of this approach lies in the view that this weather modification activity operates only to

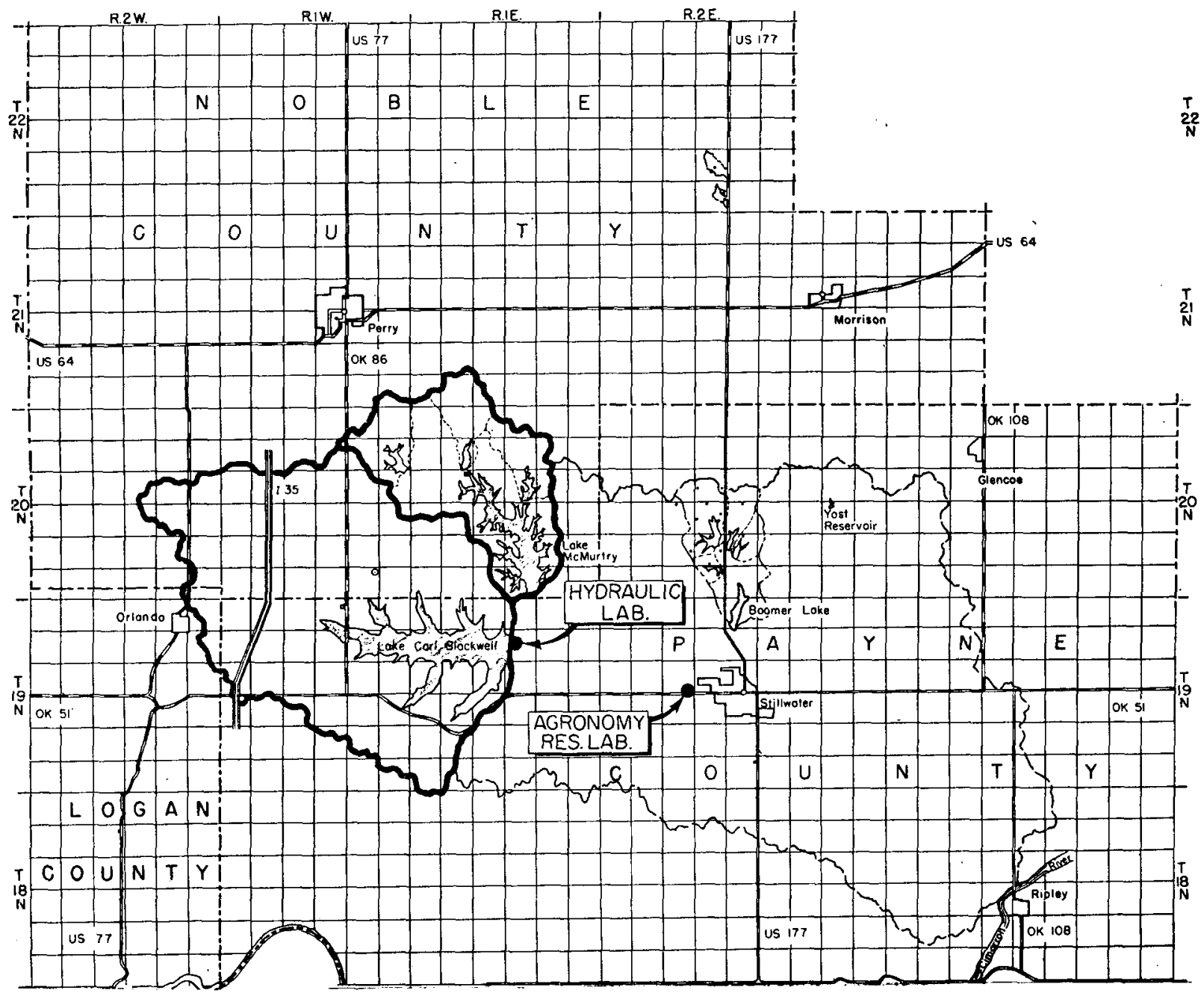

FIG. 2. Map of watershed areas for Lakes Carl Blackwell and McMurty in western Payne County, Oklahoma. 
improve the rainfall gradient of the targeted area relative to its surroundings, all of which surroundings then become controls upon the performance of the experiment, not in time, but rather in space domain. However, the special requirements of uniformity in meteorology limit such an approach to a few selected areas. Stillwater, Okla., is fortuitously situated in such an area, and is, therefore, used to illustrate the proposed method. The question of benefit to the target area at the expense of the surrounding non-target areas is not answered by this method. However, successive or enlarged applications of this method to the surroundings can give some idea as to absolute behavior of that area.

\section{The Stillwater experiment}

The City of Stillwater currently obtains its water supply from one reservoir, Lake Carl Blackwell, which is owned and operated by Oklahoma State University. The second reservoir, adjacent to Lake Carl Blackwell and called Lake McMurtry, has been built by the City and is presently awaiting enough water to become operational as a second source of supply. Fig. 2 shows a map of the two watersheds with respect to the City of Stillwater. That map also locates the two raingages which are of importance in this study as representing the target rainfall.
The regular raingage reported as the Stillwater $2 \mathrm{~W}$ gage in the ESSA Climatological Data of Oklahoma is located $2 \mathrm{mi}$ west of city-center at the Oklahoma State University Agronomy Research Station. A second raingage, with considerable data, is located at the spillway of the Hydraulics Laboratory at Lake Carl Blackwell and is $8 \mathrm{mi}$ west and $1 \frac{1}{2} \mathrm{mi}$ north of city-center.

Because of recent drought conditions in Oklahoma, as well as in other states, the Lake Carl Blackwell water supply was in critically low condition. In the spring of 1972 a joint ad hoc committee, including the mayor, the city manager, a city commissioner, a University VicePresident and several University personnel, including this author, and others, met to discuss the problem. It was decided that the only short-term remedy available was that of attempted weather modification.

After consulting with three operational weather modification people, one company was selected to help alleviate the drought. Irving P. Krick \& Associates, incorporated in Texas as Water Resources Development Corporation, was selected to seed with silver iodide generators located in the State with Stillwater as the target. Those generators are all ground-based arcburners and number a total of twelve located as shown in Fig. 3. The target area is specifically the watershed areas of Lakes Carl Blackwell and McMurtry, shown outlined in Fig. 2; but, more realistically, the target

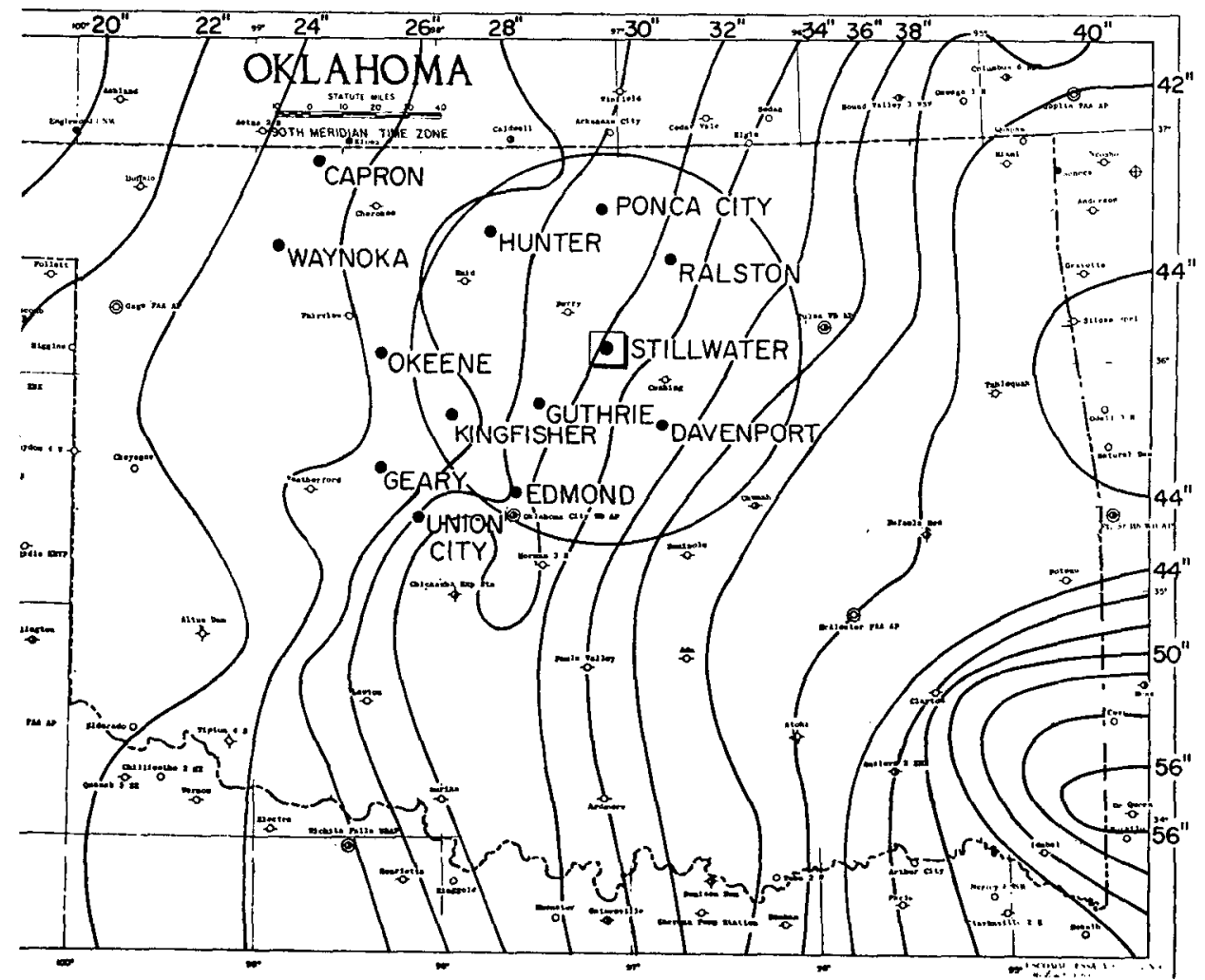

FIG. 3. Map of north central Oklahoma showing the designated target-the Lake Carl Blackwell watershed -together with neighborhood raingage stations and 30-year average isohyets for the region. 


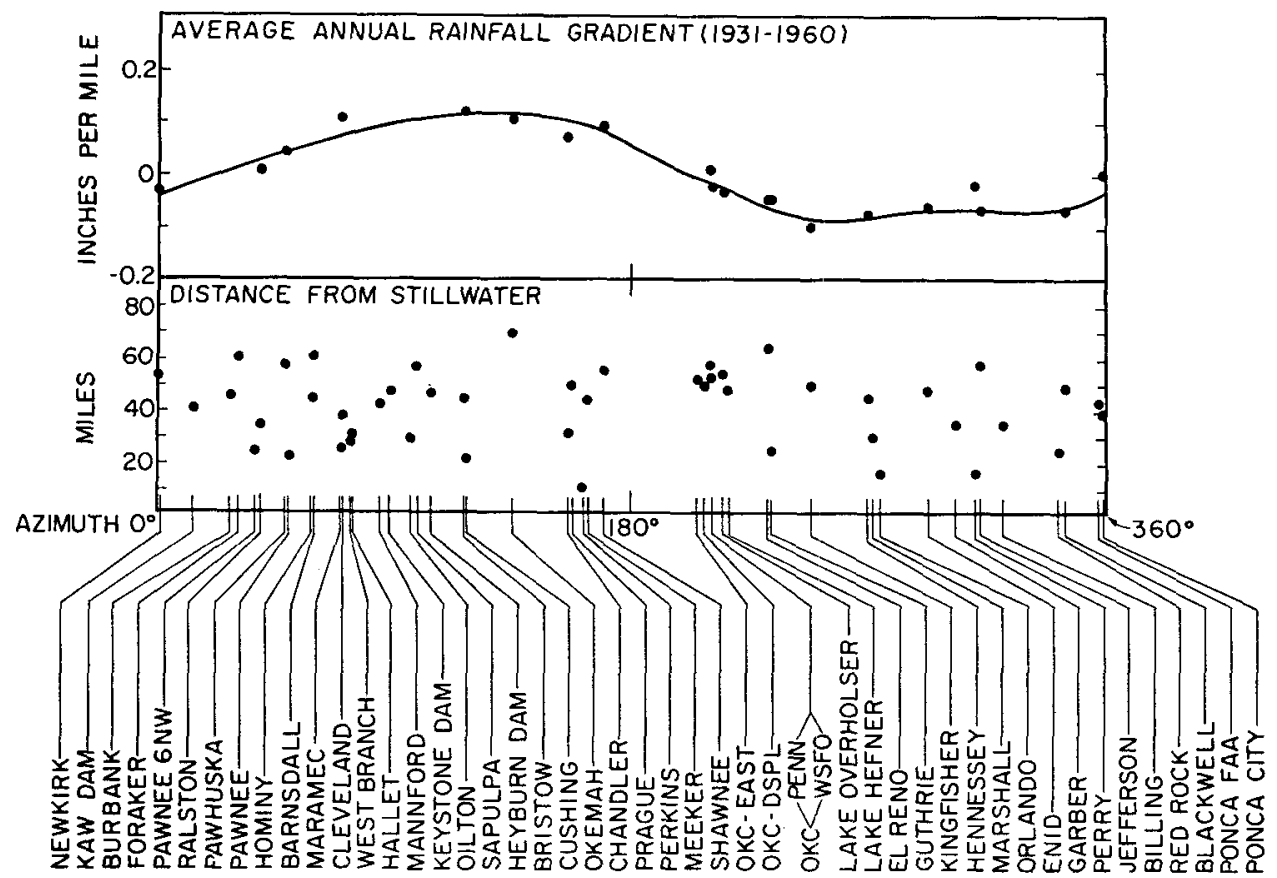

FIG. 4. Plots of mean annual precipitation gradient along a 60 -mi radius circle centered upon Stillwater (top), station distances from Stillwater (center), and National Weather Serivce raingage reporting stations plotted according to their respective azimuths from Stillwater (bottom).

area is western Payne County and immediately adjacent parts of Noble, Logan and Lincoln counties.

The claim of the Water Resources Development Corporation is to change the existing rainfall gradient to favor the target area over a period of time not less than one year but certainly by the end of the five-year operational contract time.

The methodology presented herein thus represents the first step in a consumer's attempt to know whether or not the performance of the contractor meets both contract requirements and the consumer's expectations. At the same time, both economic and scientific constraints are rather severe on the evaluation. That is, only regularly available U. S. Weather Bureau raingage data are available for the evaluation. No specialized equipment is àvailable nor have extra raingage stations been installed or considered. No formal funding of the evaluation has been forthcoming.
In addition, the actual delimitation between what is "target" area and what is not target is not clearly known at this time. There are no known lee waves or standing waves in this (Stillwater) region, but the nocturnal jet does influence distribution of airborne matter. Therefore, it is one further task of this method of evaluation to try to map or otherwise determine the actual extent of the target in all directions from the area officially designated as "the target."

\section{Data and analysis \\ a. Defining the neighborhood}

Fig. 3 shows an enlarged section of the rainfall contour map from the ESSA Climatic Atlas. That section contains Stillwater, Okla., and shows a 60-mi radius circle drawn with its center upon Stillwater. One can see that the isopleths of rainfall are quite regular in the eastern

TABLE 1. Stillwater annual precipitation (inches) using the raingage now known as Stillwater $2 \mathrm{~W}$.

\begin{tabular}{|c|c|c|c|c|c|c|c|c|c|c|}
\hline \multirow[b]{2}{*}{ Decade } & \multicolumn{10}{|c|}{ Years } \\
\hline & 0 & 1 & 2 & 3 & 4 & 5 & 6 & 7 & 8 & 9 \\
\hline 1890 & & & & & 28.24 & 29.85 & 31.40 & 31.58 & 42.62 & 32.87 \\
\hline 1900 & 29.98 & 19.98 & 40.29 & 32.58 & 31.35 & 38.59 & 41.55 & 36.65 & 61.10 & 31.76 \\
\hline 1910 & 18.22 & 34.43 & 27.60 & 36.59 & 16.79 & 48.02 & 28.01 & 24.66 & 39.85 & 31.16 \\
\hline 1920 & 47.44 & 37.10 & 34.80 & 45.39 & 23.98 & 22.44 & 32.73 & 38.10 & 33.69 & 38.45 \\
\hline 1930 & 26.05 & 30.90 & 39.08 & 35.75 & 30.67 & 33.59 & 18.29 & 25.49 & 35.29 & 26.95 \\
\hline 1940 & 33.93 & 43.68 & 45.33 & 31.07 & 31.24 & 34.03 & 28.30 & 27.40 & 31.64 & 30.24 \\
\hline 1950 & 22.80 & 34.67 & 24.12 & 32.71 & 18.33 & 27.98 & 16.68 & 48.59 & 31.85 & 61.87 \\
\hline 1960 & 35.99 & 38.89 & 32.43 & 27.14 & 26.56 & 27.78 & 25.39 & 31.48 & 33.04 & 27.84 \\
\hline 1970 & 28.68 & 31.45 & 28.12 & & & & & & & \\
\hline
\end{tabular}




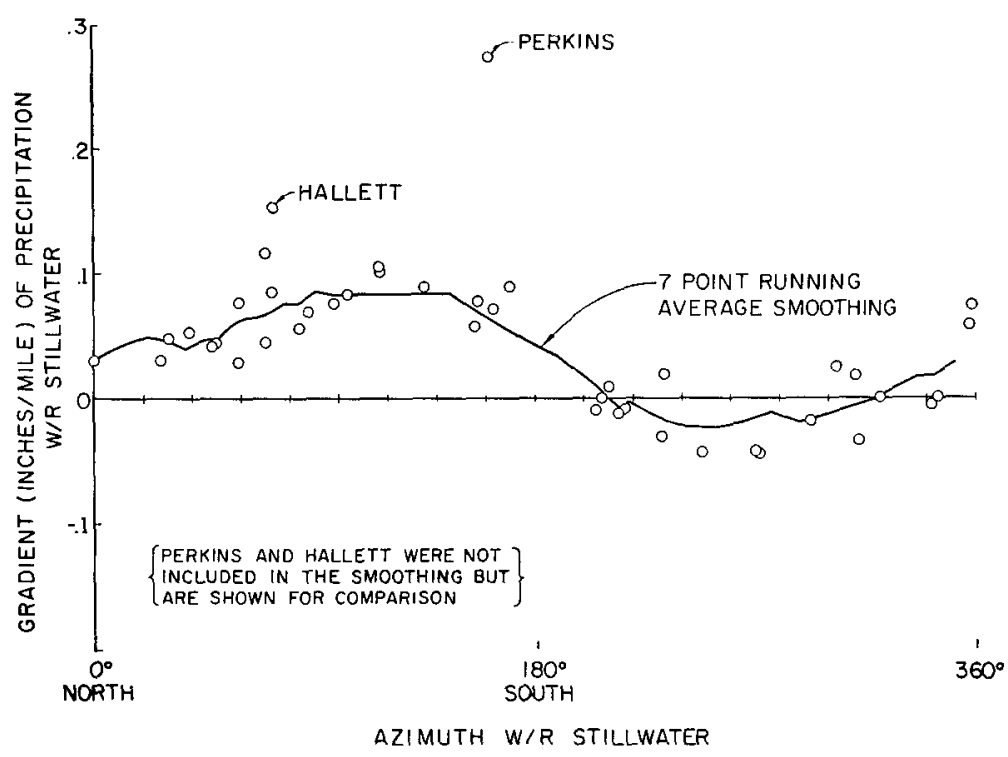

Fıg. 5. Plot of smoothed and individual points of neighborhood gradient profile based upon data from years 1950 through 1971, excluding 1959, and excluding Hallett and Perkins in the smoothing.

half of the circle while not quite so much so in the western half. Within that circled area the gradient is relatively flat when compared to other regions east and west. Radial geometry within the general area of the circle will be used to analyze and define the neighborhood of Stillwater.

Fig. 4 shows two plots and a listing of stations considered in this paper. The upper plot shows the smoothed mean gradient of precipitation in the neighborhood of Stillwater (the solid line) as well as the actual gradients (points) of the 20 stations with data extending from 1931 to 1960. The center plot shows the distance of each listed station from the Stillwater $2 \mathrm{~W}$ raingage. The list of 48 stations are all possible present weather service stations within approximately $60 \mathrm{mi}$ of Stillwater. Several among this list will not be used for reasons to be stated below.

Plotting the data in this manner has two advantages. The first, and primary, advantage is that one may judge the gradient behavior of any one station with respect to its neighbors. It happens that a few stations ${ }^{1}$ have consistent, anomalous behavior and should not be used as controls. A station may have a poorly representative sample of precipitation, or a faulty raingage, for one year, and should not be considered for that year. So this plot is useful for editing of non-representative data.

Second, the plot can give a picture of departures from normal in various directions or locations with respect to Stillwater. This factor will be important in the

1 Perkins and Hallett stations both exhibit high correlations of rainfall with Stillwater but also exhibit consistently anomalous rainfall amounts with respect to their neighbors. Therefore, these stations will not be considered as controls in this paper (see Fig. 5). determination of upwind or downwind or plume behavior outside the designated target area.

The graph shows what one would expect by looking at the contour map. Expected average rainfall is greater southeastward from Stillwater (maximum gradient occurs at $130^{\circ}$ and is a plus 0.1 inch per mile average at the 60-mi distance compared to Stillwater) and is reflected in the large positive gradient of Fig. 4. Expected annual rainfall is less westward from Stillwater with a more irregular gradient than toward the east.

\section{b. Forming the statistics}

The next step is to form the usual statistics: the mean $\bar{P}_{x}$ of annual precipitation at any station $x$; the variance and standard deviation, $\sigma_{x}{ }^{2}$ and $\sigma_{x}$, respectively, over the years of data at station $x$; and the correlation coefficients $R_{x y}$ between stations. In performing this analysis, data from the years $1950-71$ have been chosen because they were readily available at the library, and the time period is more recent. Therefore, the number of stations with sufficient data is greater than that from the period going back to 1931 (the initial date of the National Weather Service statistical data base).

Therefore, from this point onward, the data will be for the years 1950-71. Also of note is the fact that 1959 was an abnormally heavy rainfall year at the Stillwater raingage. Therefore, data from the year 1959, rather than being edited for the particular time of excessive rainfall at all stations, have been completely deleted for that year, making the data base effectively 21 years in number. Henceforth that will be the case unless otherwise stated. 


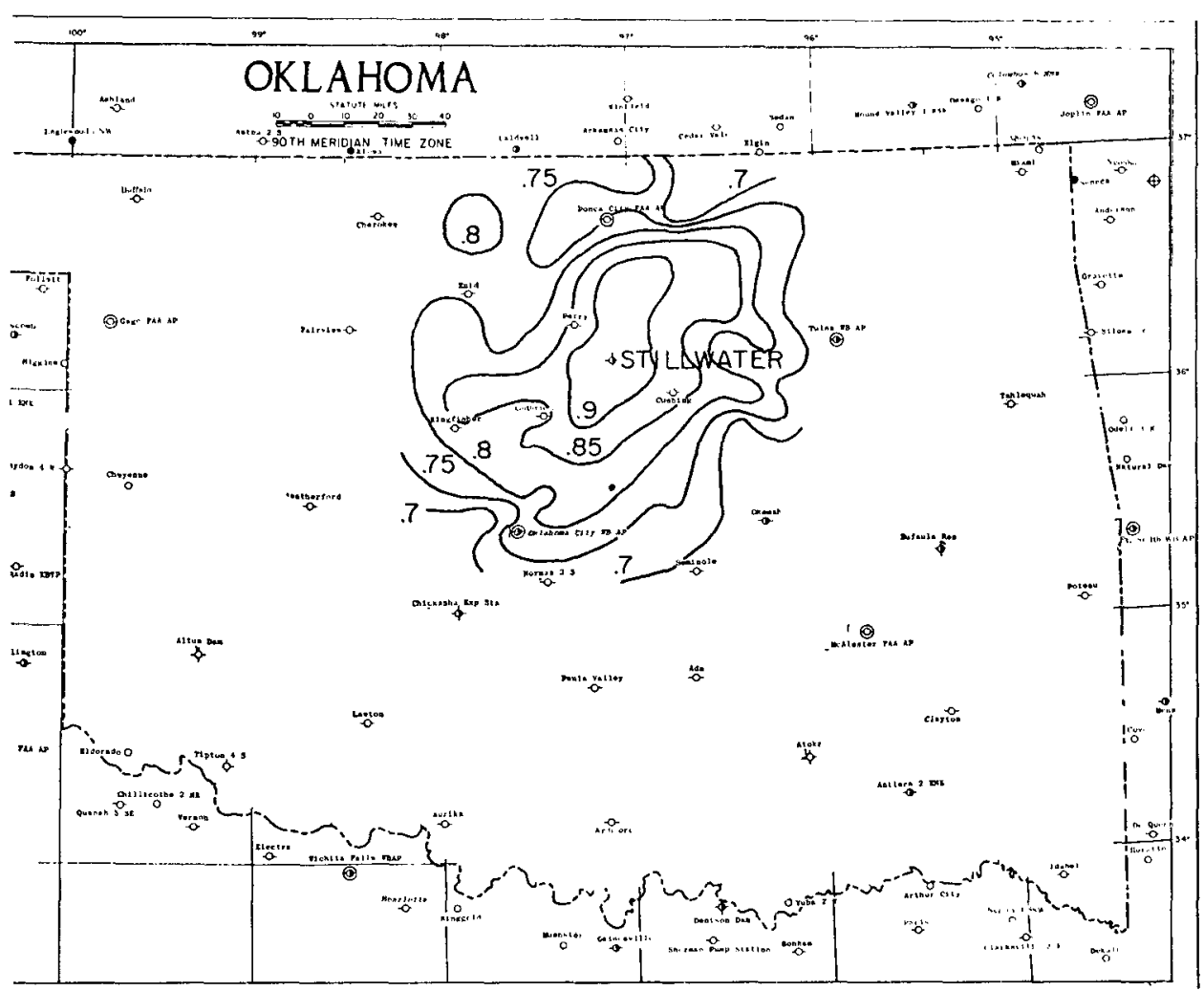

FIG. 6. Annual precipitation correlation coefficient map for the neighborhood of Stillwater based on annual precipitation during the years 1950 through 1971, excluding 1959, and taken one year at a time.

Table 1 gives the Stillwater annual rainfall data for all years 1894 through 1972 . These data, together with the corresponding annual precipitation for each of 42 acceptable of the surrounding 48 stations, are used to form the basic statistics.

To be acceptable, the station must: 1) have continuous data since 1950, with no more than one year of missing data in the years 1950 through 1971; and 2) show a gradient behavior such that the mean gradient is within one standard deviation of each nearest neighbor on each side.

Using these two criteria, six of the total of 48 possible stations in the defined neighborhood were deleted. Pawnee 6 NW, Oilton, Orlando and Kaw Dam have insufficient and unreliable data and were therefore deleted by Criterion 1. Perkins and Hallett stations, while each have acceptable quality and quantity of data, fail by criterion 2. That behavior is illustrated in Fig. 5. Thus, 42 acceptable stations remain and were used.

Fig. 5 shows the newly formed and smoothed gradient profile similar to that of Fig. 4 except derived from the data base of years 1950 through 1971 and excluding 1959, while Fig. 6 shows a correlation coefficient map for the neighborhood of Stillwater for these same data base years. Each year's gradient was taken individually, i.e., not averaged, for the calculations. The correlation coefficients for use in Fig. 6 are functions of two variables, space and time:

$$
R_{x, s w o}(d, t),
$$

where $d$ is the distance between the two stations. Thus, from a definition of the correlation coefficient, it is required that the covariance $\sigma_{x, s w o}$ also be a function of both space and time, i.e.,

$$
\sigma_{x, s w o}(d, t)=R_{x, s w v}(d, t) \sigma_{x}(l) \sigma_{s w v}(t) .
$$

The precipitation at each station raingage can be considered to be infiuenced by two factors. The first factor can be viewed as a "common mode" factor. That is, if it is raining in the neighborhood, then all gages in the neighborhood will likely receive some precipitation. ${ }^{2}$ The second factor is a random sampling factor and depends upon the sampler (gage) location, the precipitation distributions, and other such (largely unknown) factors.

The strong $(\geqslant 0.7)$ correlation coefficients in the neighborhood of Stillwater suggest that up to one-half the "common mode noise" in precipitation statistics can be cancelled or minimized by differencing the data between stations. The map of Fig. 6 also suggests there

\footnotetext{
${ }^{2}$ Data stratification into frontal and air mass situations, which show different degrees of coherence, would help define "likely."
} 


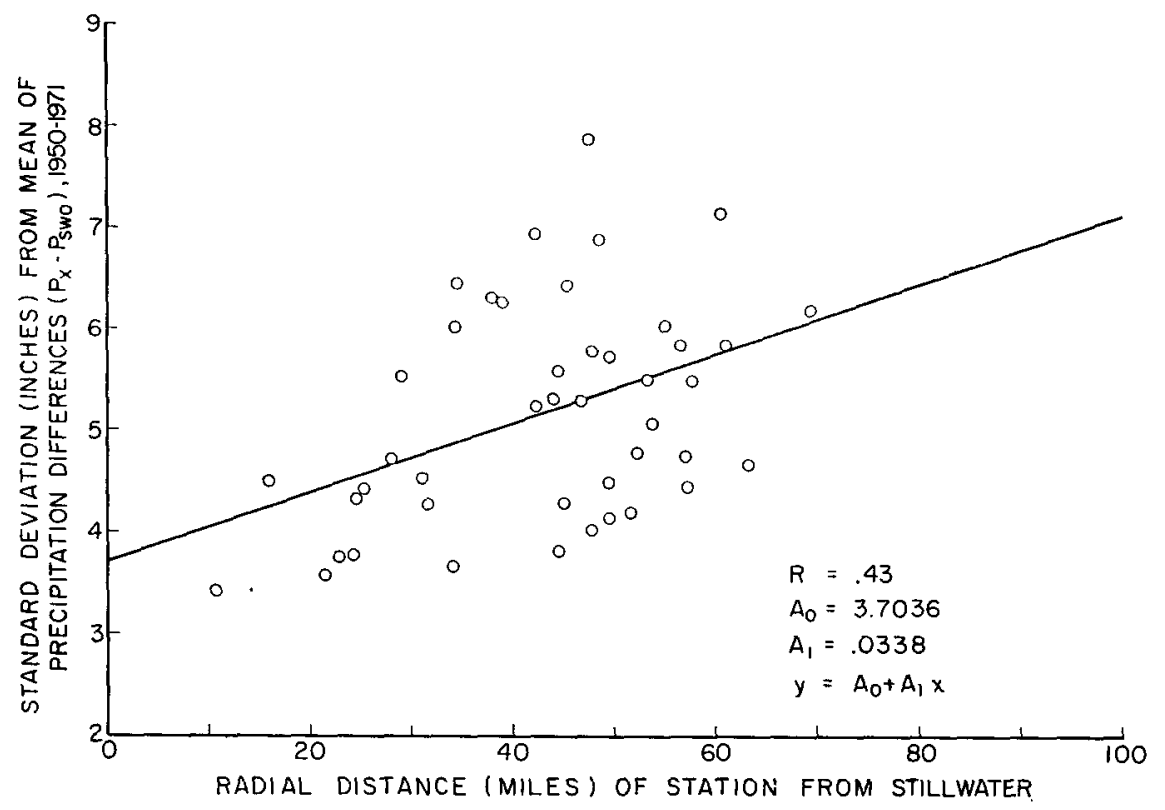

FIG. 7. Linear regression of variance in precipitation difference vs radial distance from Stillwater for 44 stations of interest in the neighborhood of Stillwater.

is an elliptical symmetry to this common mode noise effect.

After performing this differencing on the statistics, it is of interest to see whether there remains a distance effect or whether time variations in the precipitation are important.

Thus, $\Delta P_{x-s w_{0}}$ is formed for the difference in precipitation between station $x$ and Stillwater for each station and for each year of the selected time interval. A variance (standard deviation) is calculated for each of the 42 sets of station differences of interest using the 21 years of precipitation differences for that set.

One would now expect that the resulting variances would increase as a function of increasing distance from Stillwater. The plot of regression of standard deviation in $\Delta P_{x-s w o}$ against radial distance from Stillwater (Fig. 7) shows, however, that the change in distance accounts for only a minor contribution (about 18\%) to the total variance of precipitation difference. The implication is that the time, i.e., the year-to-year variance in precipitation, accounts for a much larger contribution to total variance than does distance change. The smallest correlation coefficient in this area is 0.7 , indicating that not less than $50 \%$ of the "noise" in the data is common mode noise, that is, common mode variance in the precipitation. Of the remainder, time variance contributes the most to the variance behavior.

This result indicates that comparison of differences in precipitation between stations over a given (yearly) interval will result in substantially lower variances than will comparison of year-to-year precipitation at any one or several locations.
Following this result, one observes that differential precipitation analysis should be used as a test parameter, and differences in precipitation between stations should be formed as the basic data set upon which to form variances and perform other statistical tests for weather modification. This is true especially when one wishes to observe spatial changes, i.e., has "target" and "non-target" areas. The behavior difference between target and non-target areas should then emerge (or fail to emerge for null results) as a relatively strong correlation in otherwise weakly correlated events.

\section{c. Gradient analysis applied}

Since the stations in the defined neighborhood of Stillwater are not at a uniform distance but are distributed in approximately a Gaussian manner, radially in distance with respect to Stillwater, it is necessary to form the gradient of precipitation rather than just precipitation difference for each station. Use of precipitation gradient allows comparisons among stations and in various directions and distances from stillwater throughout the whole area of the neighborhood, not just at specified radii from Stillwater.

Fig. 8 shows the 22-year period (excluding 1959) average smoothed gradients together with the $\pm 1 \sigma$ (one standard deviation) of the gradient over the 21 data years taken one year at a time (solid lines), averaged using two years at a time (dashed lines), and averaged using three years at a time (dotted lines). That is, the basic one-year gradients are then averaged over two years and again over three years to yield running mean gradients. The variances to those running mean gradients are then shown in Fig. 8 as the "2-years 


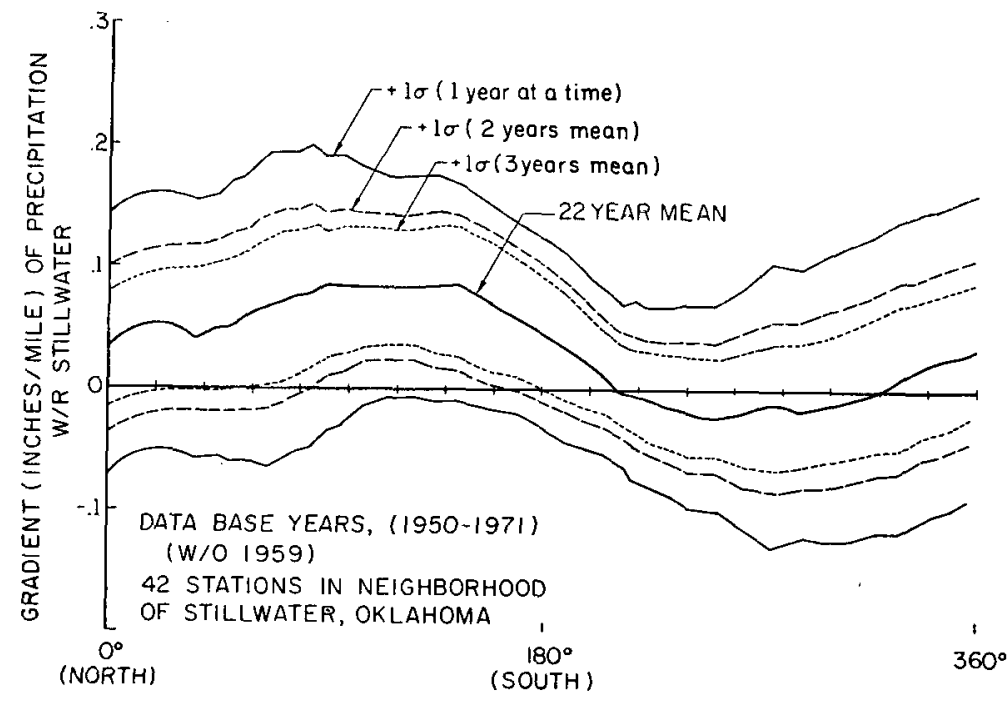

AZIMUTH W/R STILLWATER

Jir. 8. Smoothed 21-year precipitation gradients with respect to Stillwater, showing $\pm 1 \sigma$ (one standard deviation) to the mean gradient when the 21 years of data are averaged one year at a time (solid), two years at a time (dashed) and three years at a time (dotted).

mean" and the "3-years mean" standard deviations. It is of passing interest to note that the variance (and mean variances) of the gradient of precipitation is a minimum between Stillwater and the National Weather Service Oklahoma City Station, OKC-WSFO. This behavior is almost as true for OKC-Penn and OKCEast, in that order.

The implication is that, compared to all other stations in the neighborhood, the Oklahoma City Stations show the least yearly scatter in their data. If one were to choose a reference station, he would, therefore, choose $\mathrm{OKC}-\mathrm{WSFO}$ as the best as far as data variance.

It is also of fortunate coincidence that the gradient between Stillwater and the OKC stations is almost exactly zero, on the average. Therefore, precipitation amount forecasts for $\mathrm{OKC}$ will apply, on the average, quite accurately to Stillwater.

The reduction in variance of gradient by averaging over a given number of years of data is illustrated in Fig. 9 for the stations Burbank, Pawnee, Lake Overholser and Enid, as well as the 42-station average. The gradients for each of the stations was averaged over the number of years indicated using data from the base data period 1950 through 1971 but excluding 1959. The averages were running averages in the sense that years 1950,1951 and 1952 were averaged for one data point, years 1951, 1952 and 1953 were averaged to yield a second data point, and so on, to represent the " 3 years of data" variance behavior. The mean variance behavior, then, is not based on independent samples, but rather on overlapping samples of prior history.

Given homogeneous (ergodic) data over all stations, one would expect the curves of Figs. 8-10 to behave as $\sigma \propto N^{-\frac{1}{2}}$, where $N$ is the number of years of data. However, as Fig. 9 illustrates, while that is true for the ensemble of stations, it is less true for individual stations. In this case the smoothed mean gradient is non-zero and non-constant from one station to the next, even for stations adjacent to each other; thus, it's perhaps misleading to try to directly compare one station's variance behavior with another's behavior without first normalizing the data. But also of equal importance, there may be cyclic or harmonic behavior in time in the data. Therefore, the averaging process, which is a form of spectral filtering, may suppress or accentuate the effect depending upon the number of years averaged. This fact should, perhaps, be investigated further.

Fig. 10 presents a typical, but worse than average, precipitation gradient variance behavior in the defined neighborhood as a function of years of data available. Also shown on the graph is a simulated change in the "target" (Stillwater) rainfall. The target's change is assumed to be a fixed percentage and uniform over the number of years shown.

The purpose of Fig. 10 is to illustrate the number of years of data necessary to observe a given percentage change in the target's rainfall with respect to another (control) station. Thus, for example, to observe change of $\pm 10 \%$ at a level greater than $\pm 1 \sigma$ takes no fewer than three years of data. A consistent change of $\pm 5 \%$ in the Stillwater rainfall would take upward of seven years to detect at a significance of $\pm 1 \sigma$. One would hope to be able to state significance levels for any one observation at $2 \sigma$ or $3 \sigma$, but it is clearly not reasonable to do that in the time frame involved here nor, unless 


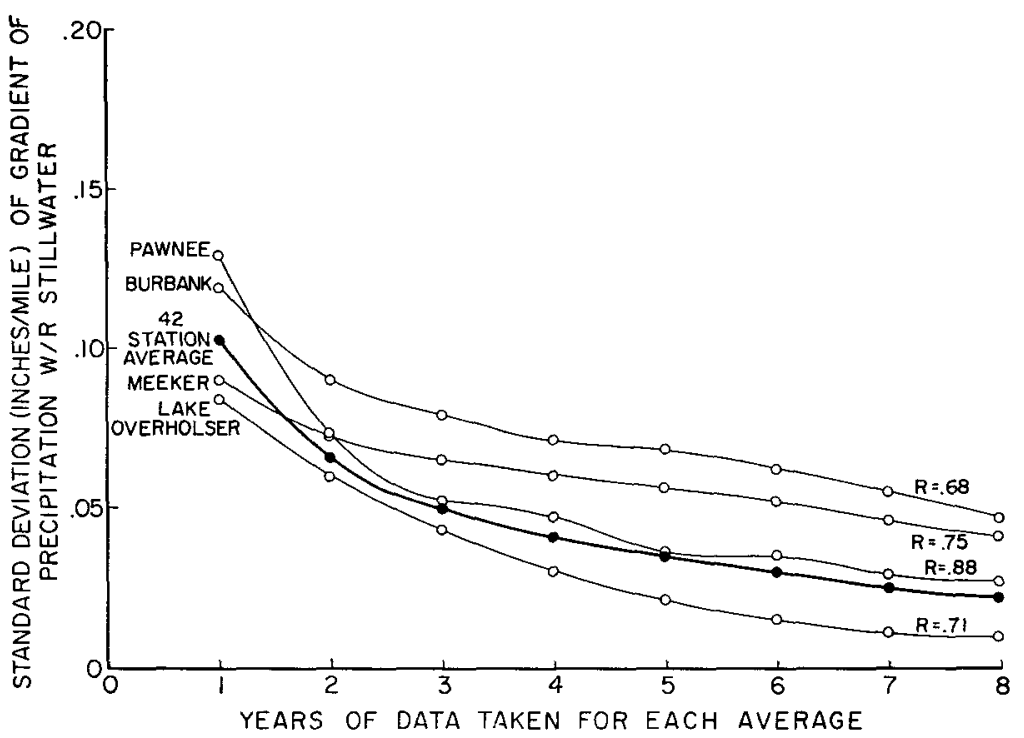

FIG. 9. Behavior of variance in mean gradient of precipitation as a function of number of years of data averaged. Four typical stations and their precipitation correlation coefficients are shown: Burbank $(R=0.68)$; Pawnee $(R=0.88)$; Lake Overholser $(R=0.71)$; and Enid $(R=0.79)$. The average behavior of 42 stations is also shown.

data stratification and other more sophisticatedmethods can be used, to minimize complicating factors. That is, the consumer must trade confidence in results for earlier (and less expensive) results.

The problem of assessing overall confidence levels

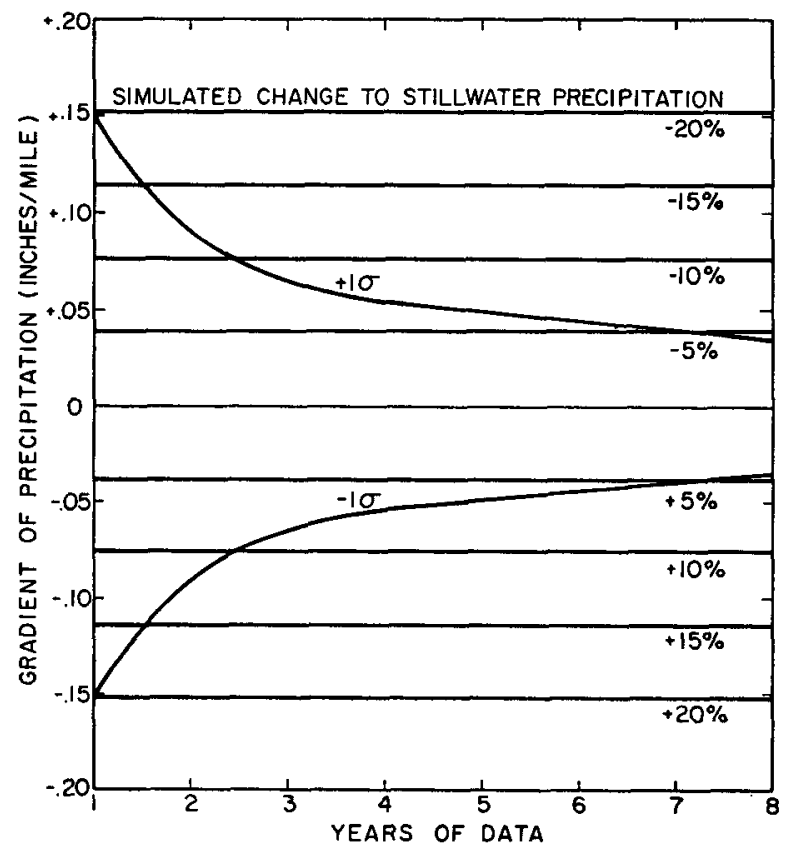

FIG. 10. Typical behavior of the variance in mean gradient of precipitation as a function of number of years of data averaged and superimposed on a simulated change in mean gradient for a given mean percentage change in precipitation at Stillwater. and significance of results now involves deciding on how many such observations need to be made. Since the correlation coefficient map (Fig. 6) indicates strong correlations in precipitation between Stillwater and all other stations in the neighborhood, it is presumed that the same type of correlations exist among all stations. Therefore, no set of data are clearly independent of any other set of data.

However, there are geometric considerations that may apply in establishing confidence levels. For example, one would expect to see a downwind effect more certainly than an upwind effect of seeding on the plains, i.e., where standing waves or mountain lee waves are not regularly observed.

Again, if weather modification contributes to an increase in rainfall (from all types of storm), one would expect continuous regular differences in precipitation throughout the year rather than one or two storms accounting for the yearly difference in precipitation between any two stations. In this case one also needs to stratify the data as to weather types, cloud top temperatures, etc. Figs. 11a and 11b show plots of data points and the resulting regression lines for the number of occasions (that the precipitation at each of the stations exceeds the precipitation at Stillwater, the target) versus the annual precipitation difference for 1967 and 1968. The regression line separates the stations which experienced positive rainfall differences more often than average (above the line) from those experiencing positive rainfall differences less often than average (below the line). Another way of looking at Figs. $11 \mathrm{a}$ and $11 \mathrm{~b}$ is that for a given difference in 

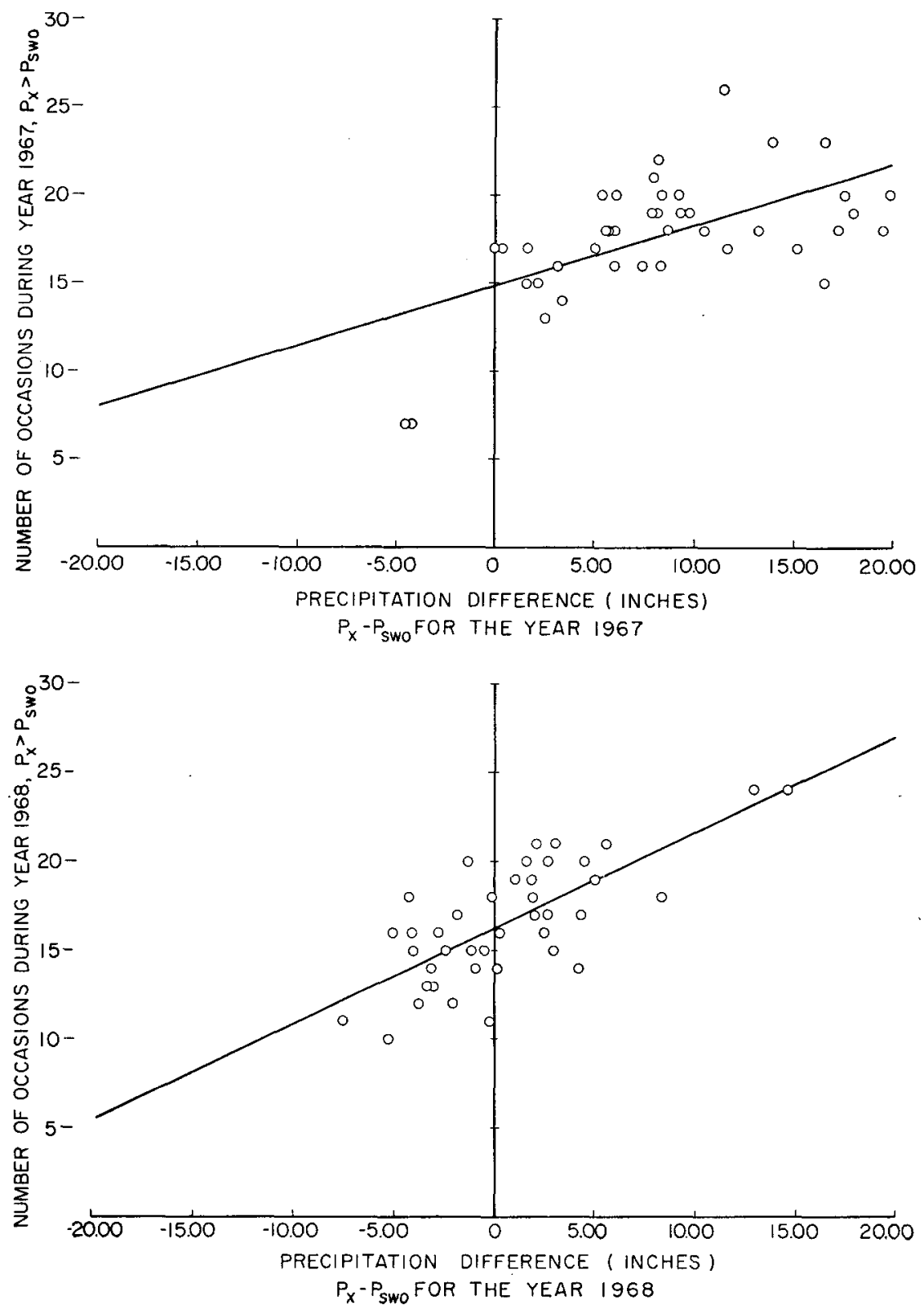

FIG. 11. Linear regression for a plot of number of occasions (during the year in which rainfall at each of 42 stations exceeds the rainfall at Stillwater) versus the rainfall difference between the station and Stillwater for 1967 (a) and 1968(b).

precipitation between Stillwater and each of the other stations one may observe whether that difference accumulated over many occasions (above the line) or occurred due to just a few very heavy downpours (below the line).

That analysis can be made for each year and can indicate the predominant rainfall regime for any station in any year compared to the neighborhood average behavior.

Perturbations at the target due to cloud seeding, when subjected to such an analysis as in Fig. 11, will show a negative change in the correlation (slope) of the regression line. The main purpose, however, of such a study is to evaluate a year's rainfall regime as to types of precipitation, i.e., a few cloudbursts or many sprinkles, compared to the control station (Stillwater).

\section{Summary}

It has been shown using correlation coefficient analysis that year-to-year variance in precipitation statistics contributes far more to the observed total variance in the Stillwater, Okla., neighborhood than 
does station-to-station variance when considered at 1-year intervals. Therefore, it is concluded that a precipitation difference or a gradient analysis, which eliminates "common mode" variance, results in a reduction of variance in precipitation statistics in that neighborhood enough to resolve small $(>10 \%)$ presumed weather modification changes within a few years $(\sim 3-5)$.

Geometry, other types of studies, and independence of data are all factors entering into the assignment of confidence levels and have only been briefly mentioned here. Further study of this problem is needed.

It is expected that at least three or four more years of data will be needed at the Stillwater location before the method of neighborhood gradients can detect a $10 \%$ benefit or decrement in precipitation at a $1 \sigma$ (15-20\%) confidence level. In an attempt to speed evaluation, subsequent use will be made of this study as it applies to already existing data of several years from another location where weather modification has been performed.

Acknoreledgments. This work was sponsored in part by funds from the U. S. Army Atmospheric Sciences Laboratory, White Sands Missile Range, N. M., under Contract DAAB07-68-C-0083.

\section{REFERENCES}

Neyman, J., H. Osborn, E. Scott and M. Wells, 1972: Re-evaluation of the Arizona cloud-seeding experiment. Proc. Nat. Acad. Sci., 69, 1348-1352.

Proceedings of the Fifth Berkeley Sym posium, 1967 : Vol. V, Weather Modification. University of California Press, Berkeley.

Schickedanz, P. T., and F. A. Huff, 1971: The design and evaluation of rainfall modification experiments. J. Appl. Meteor., 10, 502--514.

U. S. Department of Commerce (ESSA), 1968: Total Annual Precipitation (1931-1960), Climatic Atlas of the United States. Gov't Printing Office, Washington, D. C. 